Int. J. Odontostomat., 8(1):125-131, 2014.

\title{
Examen Nacional de Odontología. Revisión Bibliográfica de Instrumentos
}

\author{
Dentistry National Exam. Bibliographic Review of Mechanisms
}

Jorge Fuentes*; Mónica Silva* \& Stefano Llermaly*

FUENTES, J.; SILVA, M. \& LLERMALY, S. Examen nacional de odontología. Revisión bibliográfica de instrumentos. Int. J. Odontostomat., 8(1):125-131, 2014.

RESUMEN: Los procesos de evaluación contribuyen de manera efectiva al mejoramiento de la formación y, en consecuencia, al desempeño de los egresados en el campo laboral. La heterogeneidad de la realidad educativa actual, la diversidad de calidad de las instituciones de educación superior, entre otros factores, ameritan progresar en los sistemas de medición de competencias de egreso, asegurando entregar un profesional óptimamente capacitado al servicio de la sociedad. Si bien la evaluación de la calidad de la formación de los egresados, a través de un examen nacional, es una realidad reciente en Chile, existe experiencia internacional al respecto. Esta revisión pretende contribuir a conocer los exámenes que aplican diferentes países a sus egresados para la medición de conocimientos y competencias, las características de algunas evaluaciones; así como las políticas y organizaciones que regulan su aplicación. En el caso nacional describe la experiencia de aplicación de un examen nacional en el área de la salud en Chile, como es el caso de medicina y enfermería.

PALABRAS CLAVE: Examen nacional, examen de competencias, odontología.

\section{INTRODUCCIÓN}

Las actuales tendencias de la educación universitaria exigen a las instituciones de educación superior el diseño de estrategias y procesos orientados a incrementar la calidad de la formación, a mejorar el aprendizaje de los estudiantes, y a reducir la reprobación y el abandono, para lograr índices de aprovechamiento y de eficiencia terminal satisfactorios. En Chile estos aspectos corresponden a indicadores de eficiencia académica.

Al respecto, existe preocupación por parte de la Asociación Chilena de Enseñanza de la Odontología (ACHEO) sobre la heterogeneidad observada en los diversos proyectos educativos que existen actualmente. La importancia de este hecho, radica en la necesidad país de contar con profesionales de odontología que cumplan las competencias para proporcionar cuidados de calidad, seguros y costo-efectivos. Los criterios de calidad para las instituciones que forman odontólogos, así como las competencias generales y específicas que deben cumplir sus egresados (perfil de egreso), están definidas a nivel nacional por la Comisión Nacional de Acreditación (CNA) y son las que rigen en los procesos de acreditación por parte de dicho organismo $u$ otras agencias .

Con estos antecedentes, se permite identificar varios factores que deben conjugarse en pos del bien común. Por una parte, se debe responder a las demandas sociales con más y mejores egresados, así como lograr una incorporación exitosa de los nuevos profesionales al mercado laboral; y lo más relevante, que ellos sean capaces de asumir en forma competente la gestión del cuidado de las personas, grupos y comunidades, como un acto exclusivo del odontólogo.

Es relevante evidenciar las competencias de los egresados de la carrera de odontología mediante estrategias de evaluación adecuadas, en la cual convergen múltiples aspectos de la organización universitaria en su conjunto, con importantes repercusiones en la calidad del proceso educativo. En ésta revisión, se exponen las distintas experiencias de diversos países que han al menos intentado implementar un examen

* Departamento de Odontología Integral Adultos, Facultad de Odontología, Universidad de La Frontera, Temuco, Chile. 
odontológico, considerando que la formación en odontología exige que los egresados sean capaces de conseguir la salud bucal integral de sus pacientes, por lo que durante su formación profesional deben alcanzar esta capacidad expresada en un conjunto de competencias integradas en habilidades, actitudes, conocimientos y valores que deben evidenciarse en el examen de titulación (Beltrán \& Beltrán, 2003).

\section{Marco Referencial}

En la mayoría de los países, examen final de titulación es asumido como un instrumento de evaluación de calidad que significa parte de la formación del odontólogo. Sin embargo, en la literatura de corriente principal se evidencia que al menos en Chile, existen pocos estudios sobre los instrumentos utilizados, así como su validez. A nivel internacional (Europeo, Americano y Latinoamericano), en el área salud también se aplican este tipo de instrumentos, también con poca evidencia de la real medición de las competencias que tributan al perfil de egreso. La mayoría son instrumentos teóricos que miden conocimientos y no habilidades y hay carencia de evidencia científica sobre su validez y confiabilidad.

Distintos gobiernos, instituciones, agrupaciones profesionales, organismos no gubernamentales, organismos internacionales, entre otros, conceden a las formas de evaluar la calidad académica de los egresados en odontología y el examen de titulación tiene los importantes propósitos de informar a la sociedad acerca de la calidad en la formación académica de los nuevos profesionales, contribuir a la evaluación de la calidad de la educación superior, aportar a las instituciones información que les permita definir acciones para mejorarla, ampliar el conocimiento de que disponen las instancias gubernamentales para el establecimiento de políticas de apoyo y asignación de recursos y principalmente para informar al propio sustentante acerca del nivel de formación alcanzado.

\section{Experiencias de medición de competencias en odontología}

La experiencia internacional indica que desde hace varios años se utilizan distintos métodos para evaluar competencias, tanto para certificar la formación conducente a algún título profesional como para la reacreditación profesional, gestión, trabajo en equipo, liderazgo y desarrollo profesional continuo.

Es así que en Inglaterra, Gales e Irlanda del
Norte a través de la General National Qualifications, se otorga la titulación según las bases de las capacidades y conocimientos relevantes adquiridos durante la preparación (ofqual, 2012).

En México, un gran número de instituciones de educación superior consideran que el examen de conocimientos de licenciatura (EGEL-O), implementado por el Centro Nacional para la Evaluación de la Educación Superior (CENEVAL), permite la expresión de las competencias indispensables para la obtención del título. Es una prueba de cobertura nacional que evalúa el nivel de conocimientos y habilidades académicas de los recién egresados de la licenciatura en Odontología (CENEVAL, 2011). El EGEL permite identificar si los egresados de la licenciatura cuentan con los conocimientos y habilidades necesarios para iniciarse eficazmente en el ejercicio profesional. Esta dirigido al A los egresados de licenciatura en Odontología que han cubierto el $100 \%$ de los créditos, estén o no titulados, y en su caso, a estudiantes que cursan el último semestre de la carrera, siempre y cuando la institución formadora así lo solicite.

Al sustentante le permite: Conocer el resultado de su formación en relación con un estándar de alcance nacional, mediante la aplicación de un examen, confiable y válido, probado entodo el país. Conocer el resultado de la evaluación en cada área de conocimiento y ubicar aquellas donde tiene un buen desempeño y en las que presenta debilidades. Beneficiarse al contar con un elemento adicional de utilidad para integrarse al mercado laboral.

A las instituciones de educación superior les permite: Incorporar el Examen General para el Egreso de la Licenciatura en Odontología, como un medio para evaluar y comparar el rendimiento de sus egresados con un parámetro nacional, además del uso del instrumento como opción de titulación. Tener elementos de juicio válidos y confiables, para apoyar los procesos de planeación y evaluación curricular. Emprender acciones para mejorar la formación académica de sus egresados. Adecuar planes y programas de estudio. Aportar información a los principales agentes educativos (autoridades, organismos acreditadores y sociedad en general) de la situación de individuos y poblaciones, respecto de los conocimientos considerados necesarios para integrarse al campo laboral.

A los empleadores y a la sociedad les permite: Conocer mejor el perfil de los candidatos y de quienes inician su vida laboral mediante elementos de juicio 
confiables y objetivos, para contar con personal de calidad

El National Board Dental Examination (NBDE), es un examen nacional que presentan todos los estudiantes y profesionales de la odontología en los Estados Unidos de América como evaluación primaria sobre los conocimientos más elementales en el área. EI examen se encuentra dividido en dos partes:

La Primera Parte (NBDE I) (Joint Commission on National Dental Examination, 2013a), consiste 400 preguntas de las ciencias básicas de la medicina distribuidas en 100 preguntas por área en dos episodios de 3,5 horas, estas incluyen:

1. Ciencias Anatómicas.

2. Bioquímica-Fisiología.

3. Microbiología-Patología.

4. Anatomía Dental y Oclusión.

En la segunda parte (NBDE II) (Joint Commission on National Dental Examination, 2013a), la examinación se realiza en dos días, siendo el primero un compendio estructurado de la misma forma que la primera parte del examen (400 preguntas distribuidas en dos episodios de 200 preguntas) y el segundo día con 100 preguntas acerca de casos clínicos en el rubro odontológico. Los temas a evaluar en la primera parte son:

1. Endodoncia.

2. Odontología Restauradora.

3. Cirugía Oral y Maxilofacial/Control del dolor.

4. Diagnóstico Oral.

5. Ortodoncia/Odontología Pediátrica.

6. Manejo del Paciente.

7. Periodoncia.

8. Farmacología.

9. Prostodoncia.

El aprobar los dos exámenes es el primer requisito no solo para titularse y ejercer la odontología en los Estados Unidos, sino para poder ser considerado en un programa de especialización en el rubro. El puntaje mínimo es 75 , y este se obtiene tomando como base el promedio general de todas las personas que presentaron el mismo examen en el mismo periodo y el número de respuestas correctas de quien realiza el examen. Los odontólogos graduados en Universidades fuera de los Estados Unidos de América, deben aprobar dichos exámenes como primer paso para ser considerados en un curso de revalidación de estudios.
En Europa existe una fuerte tradición estatal que determina que son los estados nacionales los que organizan los procesos de evaluación, generalmente a través de un organismo intermedio y especializado entre el gobierno y las universidades, estableciendo estándares y procedimientos. Las evaluaciones son obligatorias y tienen gran importancia en la asignación de los recursos estatales y en las modificaciones académicas periódicas (Sanz, 2004).

En México se encuentra el Examen General de Calidad Profesional para la Odontología (EGCP-O), este examen se basa en el perfil referencial de validez del profesional de la odontología de México y manifiesta el propósito de determinar la calidad de los egresados de la carrera de odontología, a través de la valoración objetiva de sus conocimientos, habilidades y valores (López, 1998).

La experiencia sobre la evaluación educativa se realizá en Brasil desde el año 1996, a través de una examinación anual de los estudiantes en condiciones de concluir sus estudios y es de carácter obligatorio, tiene por objetivo retroalimentar los procesos en la toma de decisiones para mejorar la educación de los futuros graduados con los resultados que reflejan la realidad del país (Instituto Nacional de Estudos e Pesquisas Educacionais, 1997).

Por otra parte, en Argentina, la Comisión de Evaluación y Acreditación Universitaria (CONEAU) verifica el logro de determinados estándares de formación a través del análisis de contenidos y competencias que efectivamente disponen los estudiantes (ACCEDE), es una prueba aplicada a los alumnos cercanos a la graduación y cuyo objetivo consiste en obtener información cualitativa acerca de los contenidos y competencias que necesariamente los estudiantes deben haber incorporado a lo largo de su formación (Guerrini et al., 2003).

En lo concerniente al método que emplean las escuelas y facultades de odontología, se encontró que en la mayoría de los países de la comunidad económica europea los estudiantes defienden una tesis para la obtención del grado (Agencia Nacional de Evaluación de la Calidad y Acreditación, 2004), mientras que en Colombia, con los análisis de contenidos y competencias de que disponen los estudiantes se ha logrado implementar un examen estatal de calidad que permite constatar las competencias logradas por el egresado (Asociación Colombiana de Facultades de Odontología \& Instituto colombiano para el fomento 
de la educación, 2003). En Cuba, a partir de 1997 se realiza un examen estatal mediante el cual se comprueba y certifica la competencia y el desempeño profesional del graduado (Chiu, 1997). En México, se encontró que algunas instituciones en su reglamento se refieren a "requisitos de titulación" o "modalidades de titulación", sin existir una definición amplia y precisa y es frecuente que no se expliquen las características académico-pedagógicas que sustentan dichas modalidades (Facultad de Estudios Superiores Zaragoza, 2012; Universidad de Guadalajara, 2006).

Los exámenes generales de evaluación de la calidad profesional desempeñan funciones esencialmente constructivas y educativas. En este estudio, esta línea de pensamiento fue valorada de gran importancia para el perfeccionamiento del examen de titulación que en la actualidad tiene lugar en la Facultad de Odontología de la Universidad Michoacana de San Nicolás de Hidalgo, institución que a lo largo de su historia ha tenido tres perfiles para sus egresados que se mencionan en algunos documentos o trípticos de información general, pero que no forman parte de ninguna estructura curricular ni son del conocimiento de la comunidad escolar y no guardan relación con el examen de titulación que actualmente se realiza y cuyo carácter es la evaluación de las competencias (UMSNH, 1972, 2012). Sin embargo, los objetivos educacionales del currículo en odontología se formulan para responder a las necesidades, los intereses y las posibilidades de salud bucal de la comunidad, tomando en cuenta la planificación, la ejecución preventiva, curativa, interceptiva, de mantenimiento de la salud y los aspectos socioeconómicos y culturales del individuo objeto de atención, considerándolo como un ser íntegro. Se deberá promover la adquisición de conocimientos, destrezas, habilidades y valores para el logro de una formación integral, pero no está contemplado cómo constatar estas competencias al final de la carrera (López \& Lara, 2001; Federación Odontológica Latinoamericana, 2002), las que referidas al quehacer del odontólogo han sido establecidas por la FDI World Dental Federation en Sydney el año 2003 en dos niveles (Vargas \& Rivera, 2006); considerando que deberá satisfacer una serie de prerrequisitos antes de ser considerado clínicamente competente y que sea capaz de llevar a cabo su tarea dentro de parámetros clínicos actuales y aceptables. Posteriormente, podrá ser considerado clínicamente competente en las tareas de valoración y diagnóstico del examen del paciente, comunicación y educación del paciente, ética y jurisprudencia, tratamiento, emergencias médicas y práctica gerencial (López \& Lara, 2001).

\section{Experiencias de medición de competencias en Salud en Chile}

La primera experiencia en Chile de medición de competencias en egresados de carreras de la salud fue en la carrera de medicina, a través del Examen Médico Nacional (EMN). Las facultades de medicina que constituían la Asociación de Facultades de Medicina de Chile (ASOFAMECH) implementaron, a comienzos del 2000 , un examen final común para ser rendido por todos los egresados de las carreras de medicina. Durante los años 2001 y 2002 se realizaron dos versiones piloto y la primera aplicación oficial se realizó el 17 de diciembre de 2003, aplicándose anualmente desde entonces. El EMN surgió por la necesidad de contar con una observación objetiva de los conocimientos teóricos de los egresados, al momento de su titulación profesional. En parte, fue la respuesta frente a la aparición de variadas escuelas de medicina, con distintas orientaciones respecto a sus énfasis y perfiles de egreso, con el objetivo de una entrega uniforme y mensurable de los conocimientos más propios e irrenunciables, y por otro lado, se planteó como una medida tendiente a asegurar la calidad de la educación médica. Así, este examen constituye además un elemento que permite dar confianza ante la opinión pública, puesto que mide si los egresados cumplen con los objetivos definidos y acordados del perfil profesional. El EMN fue un examen voluntario creado por las facultades de medicina para seleccionar postulantes a sus programas de postítulo y durante su administración, ASOFAMECH subvencionó el costo del examen (Castellano et al., 2011).

Posteriormente surgió el Examen Único Nacional de Conocimientos de Medicina (EUNACOM), como una medición de conocimientos y habilidades clínicas, exigido para acceder a cargos médicos y programas de especialización financiados por el estado, así como para firmar convenios con el Fondo Nacional de Salud (FONASA). Dicho examen se transforma entonces en una obligación legal, cuyo beneficio es personal (cargos, becas, convenios FONASA y habilitación profesional) y los responsables del pago son los individuos. El diseño y administración de este examen está regulado por la Ley 20.261 y su diseño y administración fue encargado por el Ministerio de Salud a ASOFAMECH en agosto de 2009. El consejo de decanos de esa asociación delega la administración del examen en el director del EUNACOM, quien cuenta con la asesoría de un consejo de directores y de un consejo estadístico. 
La segunda experiencia chilena en el área de la salud fue la de enfermería, la Asociación Chilena de Educación en Enfermería (ACHIEEN) encomendó, en el año 2004, a la Comisión Examen Nacional la tarea de establecer un sistema nacional de evaluación de los egresados de las carreras de Enfermería, denominado Examen Nacional de Enfermería (ENENF), lo que significaba asumir la responsabilidad en el diseño del instrumento, en su aplicación y en la evaluación de sus resultados (Castellano et al.).

\section{DISCUSIÓN}

Una de las definiciones más utilizadas para comprender el concepto de competencia se refiere al conjunto de aptitudes que representan una combinación dinámica de atributos que describen los resultados del aprendizaje de un determinado programa o cómo los estudiantes serán capaces de desenvolverse al finalizar un proceso educativo. Se refiere a un conjunto identificable y evaluable de conocimientos, actitudes, valores y habilidades relacionados entre sí, que permiten desempeños satisfactorios en situaciones reales de trabajo, según estándares utilizados en el área ocupacional de su propio entorno (Castellano et al.; Tuning Educational Structures in Europe, 2012). Por otra parte, la definición de Kane (1992) es dinámica e interesante, ésta define la competencia profesional, para un individuo dado, como: "...el grado de utilización de los conocimientos, las habilidades y el buen juicio asociados a la profesión, en todas las situaciones que se pueden confrontar en el ejercicio de la práctica profesional".

Las actuales tendencias de la educación universitaria exigen a las instituciones de educación superior el diseño de estrategias y procesos orientados a incrementar la calidad de la formación, a mejorar el aprendizaje de los estudiantes, y a reducir la reprobación y el abandono, para lograr índices de aprovechamiento y de eficiencia terminal satisfactorios. En Chile estos aspectos corresponden a indicadores de eficiencia académica.

Según la Historia de la Ley № 20.261 (Biblioteca del Congreso Nacional de Chile, 2008), el Dr. Lorca, presidente de la ASOFAMECH, estableció que la educación y la salud, son dos componentes fundamentales del desarrollo humano tienen en los países desarrollados que adhieren al modelo de libre mercado, y Chile aspira a constituirse en uno de ellos, un tratamiento especial del máximo rigor en la aplicación de leyes, políti- cas, normas y regulaciones para asegurar la calidad del servicio a los usuarios de los sistemas de Salud y Educación. Nuestro país debe seguir trabajando en esa dirección.

La responsabilidad que asumirá la Comisión de ACHEO en el diseño, aplicación y evaluación del examen nacional de odontología (ENO) en las escuelas a lo largo del país, será posible gracias al compromiso y apoyo de todos los actores involucrados, tales como el directorio de ACHEO y su consejo asesor, el comité técnico, las unidades académicas, la Federación de Estudiantes de Odontología, los estudiantes y el Colegio de Cirujanos Dentistas.

El gran desafío entonces es confeccionar un examen nacional escrito en forma continua y sistemática; aunque sin duda se requiere avanzar también hacia la medición de competencias de los egresados en las áreas del saber hacer y del saber ser, tal como ya se realiza en otros países. Esto se puede alcanzar a través de pruebas computarizadas en ambientes simulados, con el objetivo de asegurar lo más posible una práctica segura y efectiva.

La viabilidad del ENO significará asegurar una base sólida y objetiva de evaluación independiente de los resultados del proceso de formación de las Escuelas de Odontología del país, lo que permitirá entregar información:

1. A las Escuelas para evaluar los cambios curriculares o metodológicos que ellas efectúen.

2. A los empleadores respecto de la competencia de cada egresado.

3. A los postulantes a las Escuelas.

4. A los organismos acreditadores.

5. Al público y la sociedad.

Además, este examen podría contribuir a la evaluación de los odontólogos extranjeros que ingresan para ejercer en el país, aportando un sistema estandarizado de aseguramiento de calidad a profesionales que inmigren como consecuencia de nuevos tratados internacionales, facilitando así la movilidad hacia Chile de buenos profesionales.

\section{CONCLUSIÓN}

Se cree, con firmeza, que los procesos de evaluación y acreditación contribuyen en forma efectiva 
al mejoramiento de la formación y, en consecuencia, al desempeño de los egresados en el campo laboral. Sin embargo, la heterogeneidad de la realidad educativa actual, el insuficiente porcentaje de carreras de Odontología acreditadas, entre otros factores, ameritan progresar en los sistemas de medición de competencias de egreso, como una responsabilidad social. Por tanto, todos los esfuerzos que se realicen para evaluar el cumplimiento del perfil profesional se orientan finalmente al propósito de asegurar a las personas atención odontológica de calidad.

Su objetivo entonces sería aportar a la socie- dad información que verifique el cumplimiento de objetivos cognitivos comunes a la formación de un Odontólogo y que deben estar presentes en todas las unidades académicas pertenecientes a la Asociación, conforme a los perfiles que ellas mismas han determinado y consecuentes con los criterios de acreditación establecidos. Asimismo, debería entregar a las unidades académicas el resultado de su facultad o escuela, de modo de contribuir al mejoramiento continuo de los programas de estudio. El propósito más trascendente de esta medición es asegurar que los egresados posean las competencias disciplinares y profesionales necesarias para otorgar cuidados de calidad a las personas.

FUENTES, J.; SILVA, M. \& LLERMALY, S. Examen nacional de odontología. Revisión bibliográfica de instrumentos. Int. J. Odontostomat., 8(1):125-131, 2014.

ABSTRACT: The evaluation process is very helpful to improve student education and their subsequent performance in the work environment. The diversity of the educational reality and the quality of education, among other factors, make necessary an improvement in the graduate competences measuring system, ensuring a well trained professional with proper skills in the field. Assessing the quality of education of graduates, through a national exam is only a recent practice in Chile; however, there is an international experience. This review intends to provide information of the exams that various countries apply to measure skills and knowledge, their characteristics, as well as policies and organizations regulating their implementation. It also describes the experience of implementing a national exam in the health services area in Chile, as is the case for medicine and nursing.

KEY WORDS: National exam, competency exam, dentistry.

\section{REFERENCIAS BIBLIOGRÁFICAS}

Agencia Nacional de Evaluación de la Calidad y Acreditación. Libro Blanco. Título de Grado en Odontología. Madrid, Agencia Nacional de Evaluación de la Calidad y Acreditación, 2004. Disponible en: http://www.aneca.es/ media/150344/libroblanco_odontologia_def.pdf

Asociación Colombiana de Facultades de Odontología \& Instituto colombiano para el fomento de la educación. Exámenes de calidad de la educación superior-ECAES para Odontología- 2003. Documento de fundamentación de la prueba. Bogotá, Colombia, 2003. Disponible en: http://www.acfo.edu.co/Memorias/8_eca/xtras/2003/ fd_01.pdf

Beltrán, N. R. J. \& Beltrán, A. E. D. Taxonomía de las competencias odontológicas. Revista da ABENO, 3(1):2832, 2003.

Biblioteca del Congreso Nacional de Chile. Historia de la Ley no. 20.261: crea examen único nacional de Conocimientos de Medicina, incorpora cargos que indica al sistema de alta dirección pública y modifica la ley no. 19.644. Valparaíso, Biblioteca del Congreso Nacional de Chile, 2008.
Castellano, S. A.; Tagle, V. C.; Galdames, C. L.; Riquelme, P. N.; Landman, N. C. \& Peroni, P. S. Examen nacional de enfermería en Chile: importancia y desafíos. Cienc. Enferm., 17(1):27-36, 2011.

CENEVAL. Examen Ceneval de Odontología. Características del EGEL-O. 2011. Disponible en: http:// www.ceneval.net/odontologia/que.html

Chiu, N. V.; Rodríguez, M. G. \& Alonso, P. M. E. Experiencia del examen estatal en la carrera de estomatología. Educ. Med. Super., 11(1):47-52, 1997.

Facultad de Estudios Superiores Zaragoza. Carrera de Cirujano Dentista. Opciones de Titulación. México D. F., Universidad Nacional Autónoma de México, 2012. Disponible en: http://132.248.60.110:8081/ fesz_website_2011/wp-content/dentista/titulacion/ opcionestitulacion2012.pdf

Federación Odontológica Latinoamericana. Declaraciones de la FDI: Acción contra el ejercicio ilegal de la odontología, 2002. Disponible en: http://www.folaoral.com/ resoluciones.htm 
Guerrini, V.; Pérez, R. C. \& Jeppesen, C. Evaluación integral de los procesos de acreditación de grado. Rev. Aval. Educ. Super., 8(1):113-29, 2003.

Instituto Nacional de Estudos e Pesquisas Educacionais. Exame Nacional de Cursos 1996: síntese. Brasilia, Instituto Nacional de Estudos e Pesquisas Educacionais, 1997.

Joint Commission on National Dental Examination. National Board Dental Examination, Part I, 2013 Guide. 2013a. Disponible en: http://www.ada.org/sections/ educationAndCareers/pdfs/nbde01_examinee_guide.pdf

Joint Commission on National Dental Examination. National Board Dental Examination, Part I, 2013 Guide. 2013b. Disponible en: http://www.ada.org/sections/ educationAndCareers/pdfs/nbde02_examinee_guide.pdf

Kane, M. T. The assessment of professional competence. Eval. Health Prof., 15(2):163-82, 1992.

López, C. V. Análisis del Examen General de Calidad Profesional para la odontología en México. Reencuentro, 23:76-82, 1998

López, C. V. \& Lara, F. N. Paradigmas en la práctica y formación odontológica en México. Rev. Ateneo Argent. Odontol., 40(1):34-9, 2001.

ofqual. Register of Regulated Qualifications. 2012. Disponible en: http://register.ofqual.gov.uk/

Sanz, M. El espacio europeo en la educación superior: Una convergencia necesaria. Convergencia Europea y Titulaciones con Directivas Propias. Facultad de Odontología, Universidad Complutense de Madrid, 2004. Disponible en: http://www.unizar.es/eees uz17_Conv\%2 europea $\% 20$ y $\% 20$ titul $\% 20$ con $\% 20 \bar{d}$ irectiv as $\% 20$ propias $\% 20$ Mariano\%20SANZ.pdf

Tuning Educational Structures in Europe. 2012. . Disponible en: http://www.unideusto.org/tuningeu/home.html

Universidad de Guadalajara. Reglamento General de Titulación de la Universidad de Guadalajara. Guadalajara, Universidad de Guadalajara, 2006. http:// www.secgral.udg.mx/sites/archivos/normatividad/general/ReglamentoGeneralde Titulacion.pdf

Universidad Michoacana de San Nicolás de Hidalgo (UMSNH). Reglamento General de Exámenes, 1972. http://www.umich.mx/normatividad.html

Universidad Michoacana de San Nicolás de Hidalgo (UMSNH). Facultad de Odontología, Perfil de Egreso, 2012. Disponible en: http://www.umich.mx/licenciaturacirujano-dentista.html
Vargas, P. M. L. \& Rivera, M. N. El examen de titulación en odontología como indicador de calidad profesional en la Universidad Michoacana de San Nicolás de Hidalgo, México. Educ. Med. Super., 20(3):0-0, 2006.

Dirección para Correspondencia:

Dr. Jorge Fuentes Nawrath

Depto. Odontología Integral

Manuel Montt 110, Temuco

CHILE

Email: jorge.fuentes@ufrontera.cl

Recibido : 05-10-2013

Aceptado: 07-01-2014 